\title{
An Improved Passivity-Based Control to Buck Dc-Dc Converter
}

\author{
Yanhua Xian* \\ College of Electronic Engineering \\ Guangxi Normal University \\ Guilin, 541004, China \\ *Corresponding author
}

\author{
Xiaoshu Luo \\ College of Electronic Engineering \\ Guangxi Normal University \\ Guilin, 541004, China
}

\begin{abstract}
For the nonlinear characteristic of buck dc-dc converter and high demand of dynamic quality and robustness for the converter, a passivity-based control with an adjustable parameter for buck converter is proposed. Based on the nonlinear error model of buck converter, a feedback equivalence system is obtained by the way of coordinate transformation. According to passive theory, the feedback equivalent system is to be passive by adding feedback appropriately. The proof of the passivity of closed-loop system is given. And a Lyapunov function is constructed to guarantee asymptotic stability of closed-loop system. Finally, a passive feedback control law for buck converter is derived. Simulate by designing control circuit in Pspice environment, and the simulative results show that the stability of output voltage is effectively controlled by the proposed control method. The controller has very strong robustness for disturbance caused by load change and input voltage change. And the system dynamic response is improved by adjusting the coefficient of the control law.
\end{abstract}

Keywords-passivity; feedback equivalence; buck converter; nonlinear model

\section{INTRODUCTION}

Dc-dc switching converter works at switching periodically multiple working modes. Due to the switch turn-on and turn off, the converter is discrete although each working mode is linear and continuous. As a whole, the converter is a strongly nonlinear system. Compared with the linear method based on classical control theory, the nonlinear method to control the converter is more advantageous.

Passivity theory is an analytic technique for nonlinear system control and stability analysis. Over the past ten years, passive control method has developed a lot, which is widely applied to the power electronic system such as inverters $\left.{ }^{[} 1 \sim 3\right]$, power corrector[4], motor[5], quasi-resonant converters[6], robot manipulator[7,8], etc.. Passivity method to control dc-dc converter is also studied. In [9], a controller is designed based on passivity theory for boost converter, achieving global stability in large signal operation and good disturbance robustness to the input voltage and load. For buck converters with constant-power loads, a proportional- differential controller is derived using a passivity-based technique[10]. The works in [11] for buck converter and in [12] for boost, buckboost converters, the passivity-based controllers are obtained also by building Euler-Lagrange models. The forms of controller are simple and easy to implement. In [13], a kind of passivity control scheme is derived by constructing the standard chain type structure based on an affine nonlinear model of current conduction mode buck converter. The controller has very strong robustness for disturbance caused by load change.

In this paper, an improved controller of [13] is proposed. The nonlinear error model of buck converter is built and passive. An adjustable parameter is added in the feedback control law to stabilize output voltage and current of the buck converter and to regulate overshoot, steady-state error of system performances as well. The simulation results are reported.

\section{NONLINEAR ERROR MOdel OF BUCK CONVERTER}

The schematic diagram of buck converter is illustrated in Figure 1. The equivalent series resistances of the inductor, the capacitor, and the switch, as well as the voltage drop and the cutoff current across the diode and the switch are neglected. Supposing the switch action is instantaneous, the converter will work on two different states in one switching period under continuous conduction mode. Synthesizing the circuit state equations of the two states, the general expression of the buck converter is obtained as follows

$$
\left\{\begin{array}{l}
\dot{i}_{L}=-\frac{1}{L} u_{C}+\frac{E}{L} \mu \\
\dot{u}_{C}=\frac{1}{C} i_{L}-\frac{1}{R C} u_{C}
\end{array}\right.
$$

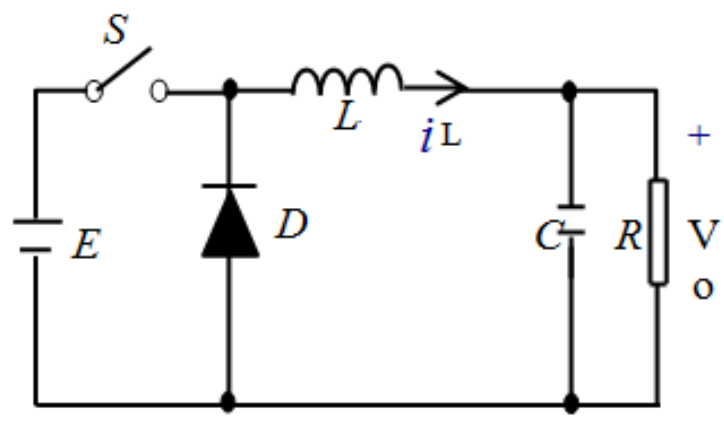

FIGURE I. BUCK CONVERTER 
where $\mu$ is a function about switch action. Switch $S$ remains turned-on from $t_{k}$ to $t_{k}+d T$, then $\mu=1$. Otherwise, switch $S$ remains turned-off from $t_{k}+d T$ to $t_{k}+T$, then $\mu=0$. T is one switching period, $d$ is the duty cycle, $0<d<1$. Define the error variables as $x_{1}=i_{L}-I_{d}, x_{2}=u_{C}-V_{d}, u=\mu-D$, where $I_{d}, V_{d}$ and $D$ are the operation point current, voltage, and duty cycle respectively. When the plant is stable, there is $\dot{i}_{L}=0, \dot{u}_{C}=0$. Then the relationship of the operation point variables can be obtained as follows: $I_{d}=D E / R, V_{d}=D E$. Putting the error variables into (1) leads to the buck converter model expressed by error variables

$$
\left\{\begin{array}{l}
\dot{x}_{1}=-\frac{1}{L} x_{2}+\frac{E}{L} u \\
\dot{x}_{2}=\frac{1}{C} x_{1}-\frac{1}{R C} x_{2}
\end{array}\right.
$$

which is control object to be passive.

\section{Passivity-Based Design}

The theoretical basis of passive control is actually the Lyapunov method. By applying appropriate feedback control under certain conditions, the closed-loop system is passive. Then construct a positive definite storage function as a Lyapunov function of the closed-loop system to ensure the asymptotic stability at zero initial state. The storage function can be derived by backstepping method. If the control object is equivalent to a series connection of an integrator and a subsystem, the Lyapunov function of the control object can be formed by adding the square of the integrator state variable and the Lyapunov function of the subsystem according to backstepping method.

\section{A. Feedback Equivalent System of Buck Converter}

The following describes the process of converting the system (2) into a series system of an integrator and a subsystem. Considering the following nonlinear system

$$
\left\{\begin{array}{l}
\dot{x}=f(x)+g(x) u \\
y=h(x)
\end{array}\right.
$$

where $x \in \mathbf{R}^{\mathrm{n}}$, $u$ and $y$ denote control input and system output respectively, the following theorems are applied.

Theorem 1[14] If there is a n-r vector function $t(x)$, $t(x)=\left[t_{1}(x) t_{2}(x) \cdots t_{n-r}(x)\right]^{T}$, satisfying

$$
L_{g} t_{i}(x)=\frac{\partial t_{i}}{\partial x_{1}} g_{1}(x)+\frac{\partial t_{i}}{\partial x_{2}} g_{2}(x)+\cdots+\frac{\partial t_{i}}{\partial x_{n}} g_{n}(x)=0
$$

where $g(x)=\left[\begin{array}{llll}g_{1}(x) & g_{2}(x) & \cdots & g_{n}(x)\end{array}\right]^{T}$, the system (3) with the relative order $\mathrm{r}(\mathrm{r}<\mathrm{n})$ is equivalent to the system as follows

$$
\left\{\begin{array}{l}
\dot{z}=\tilde{f}\left(z, y_{1}, y_{2}, \cdots, y_{r}\right) \\
\dot{y}_{1}=y_{2} \\
\quad \vdots \\
\dot{y}_{r}=v
\end{array} .\right.
$$

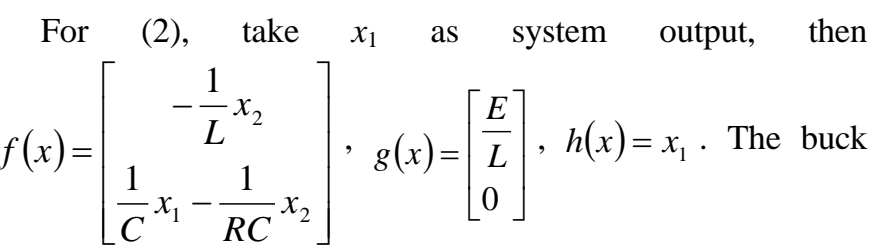
converter model (2) can be formed as (3). The relative order of the buck converter is 1 , which can be calculated by $L_{g} h(x)=\frac{\partial h}{\partial x} g(x)=E / L \neq 0$. According to theorem 1, find a one dimensional vector function $t(x)$ to meet the condition $\frac{\partial t(x)}{\partial x_{1}} \frac{E}{L}=0$. We choose $t(x)=k x_{2}$, where $k$ is an adjustable parameter. By coordinate transformation $\left[\begin{array}{l}z \\ y\end{array}\right]=\left[\begin{array}{l}t(x) \\ h(x)\end{array}\right]$, then

$$
\left\{\begin{array}{l}
\dot{z}=-\frac{1}{R C} z+\frac{k}{C} y \\
\dot{y}=-\frac{1}{L k} z+\frac{E}{L} u
\end{array}\right.
$$

Denote $\quad u=\frac{L}{E} v+\frac{Z}{E k}$

where $v$ is new control input, thus the feedback equivalent system of (2) is deduced to (6) as follows.

$$
\left\{\begin{array}{l}
\dot{z}=-\frac{1}{R C} z+\frac{k}{C} y \\
\dot{y}=v
\end{array}\right.
$$

Distinctly, the (6) is a series connection of the integrator $y$ and the subsystem $\mathrm{z}$ as shown in Figure 2.

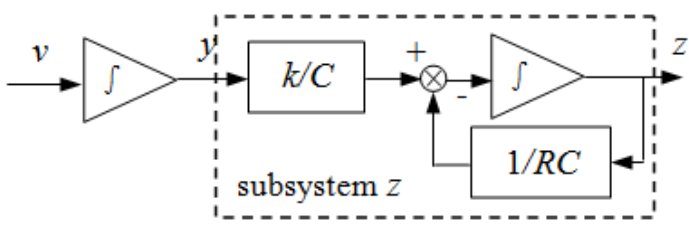

FIGURE II. SERIES CONNECTION OF AN INTEGRATOR AND A SUBSYSTEM 


\section{B. Passivity-Based Controller}

Denote $y=\beta(z)=-z$, choose a positive definite function as $W(z)=\frac{1}{2} z^{2}$. Then $\dot{W}(z)=z \dot{z}=-\frac{1}{R C} z^{2}-\frac{k}{C} z^{2}$. As $k>0$ and $z \neq 0, \dot{W}(z)<0$. Thus the subsystem $\dot{z}=-\frac{1}{R C} z+\frac{k}{C} y$ is stable asymptotically at $\mathrm{z}=0$ when $y=\beta(z)$. We choose a new output variable denoted as $\tilde{y}=y-\beta(z)$ for system (6) and make coordinate transformation from $[z y]$ to $[z \tilde{y}]$. The system (6) then can be expressed as

$$
\begin{array}{ll} 
& \left\{\begin{array}{l}
\dot{z}=-\frac{1+R k}{R C} z+\frac{k}{C} \tilde{y} \\
\dot{\tilde{y}}=v-\frac{1+R k}{R C} z+\frac{k}{C} \tilde{y}
\end{array}\right. \\
\text { Letting } \quad v=\frac{1+R k}{R C} z-\frac{k}{C} \tilde{y}+\tilde{v}
\end{array}
$$

where $\tilde{v}$ constitutes an auxiliary input signal, then (7) is rewritten as

$$
\left\{\begin{array}{l}
\dot{z}=-\frac{1+R k}{R C} z+\frac{k}{C} \tilde{y} \\
\dot{\tilde{y}}=\tilde{v}
\end{array}\right.
$$

Obviously, the output $\tilde{y}$ of (9) satisfies the condition of zero-state detectable property and the system (9) is a minimum phase system. We can get the conclusion as theorem 2 .

Theorem 2 If system (9) is a minimum phase system, there is a static state feedback as the form (10) such that closed-loop system is passive.

$$
\tilde{v}=-\frac{k}{C} z-\tilde{y}
$$

Proof Letting $v^{\prime}=-\tilde{y}$ as an input signal, the closed-loop system consisting of (9) and (10) can be described as

$$
\begin{aligned}
& \dot{\bar{x}}=F(\bar{x})+G(\bar{x}) v^{\prime} \\
& \tilde{y}=H(\bar{x})
\end{aligned}
$$

where $\bar{x}=[z \tilde{y}]^{T}, F(\bar{x})=\left[\begin{array}{c}-\frac{1+R k}{R C} z+\frac{k}{C} \tilde{y} \\ -\frac{k}{C} z\end{array}\right], G(\bar{x})=\left[\begin{array}{l}0 \\ 1\end{array}\right]$, $H(\bar{x})=[01] \bar{x} 。$

Construct a storage function $V(z, \tilde{y})=W(z)+\frac{1}{2} \tilde{y}^{2}$, yielding that

$$
\begin{aligned}
\dot{V}(z, \tilde{y}) & =z\left(-\frac{1+R k}{R C} z+\frac{k}{C} \tilde{y}\right)+\tilde{y}\left(-\frac{k}{C} z-\tilde{y}\right) \\
& =-\frac{1+R k}{R C} z^{2}-\tilde{y}^{2} \\
& \leq-\tilde{y}^{2}=\tilde{y} v^{\prime} \quad \forall t \geq 0
\end{aligned}
$$

By integral operation on both side of (11), it has (12) which is set up to arbitrary $v^{\prime}$.

$$
V(z, \tilde{y})-V\left(z_{0}, \tilde{y}_{0}\right) \leq \int_{0}^{T} \tilde{y} v^{\prime} d \tau \quad \forall T \geq 0
$$

where $z_{0}, \tilde{y}_{0}$ are initial states of the closed-loop system. Obviously, the (12) meets the definition of passivity. So the closed-loop system is passive. The proof is completed.

From (11), we can know that $\dot{V}(z, \tilde{y})<0, \forall \tilde{y} \neq 0$. It means that the storage function $V(z, \tilde{y})$ is a Lyapunov function of the closed-loop system. Thus the closed-loop system is asymptotically stable. Because the coordinate transformation from $[z y]$ to $[z \tilde{y}]$ is full rank, the feedback controller can stabilize the system (9) as well as system (6). Therefore, put (10) into (8), that is

$$
v=\left(\frac{1}{R C}-\frac{k}{C}-1\right) z-\left(\frac{k}{C}+1\right) y .
$$

According to (5), the controller for buck can be described as

$$
u=\frac{1}{E}\left(\frac{L}{R C}-\frac{L k}{C}-L+\frac{1}{k}\right) z-\frac{L}{E}\left(\frac{k}{C}+1\right) y
$$

Substituting $z=k x_{2}$ and $y=x_{1}$ in (13), the controller expression of (13) is rewritten as

$$
u=\frac{k}{E}\left(\frac{L}{R C}-\frac{L k}{C}-L+\frac{1}{k}\right) x_{2}-\frac{L}{E}\left(\frac{k}{C}+1\right) x_{1} .
$$




\section{CiRCUIT Simulation}

In order to explore the feasibility of the proposed method in the actual buck dc-dc converter, we use the Pspice circuit simulation tool to design the circuit structure of the passive controller, as shown in Figure 3. The amplifier U1A and U2A are all connected as subtraction operational amplifiers. Their input signals are output voltage and inductor current respectively sampling from the buck converter. Their outputs are the error signals about the reference voltage and reference current. The amplifier U3A and U4A are used to scale up the error signals by adjustable resistances $R_{10}, R_{14}$. The amplifier $\mathrm{U} 5 \mathrm{~A}$ is as a adder to output the control signal.

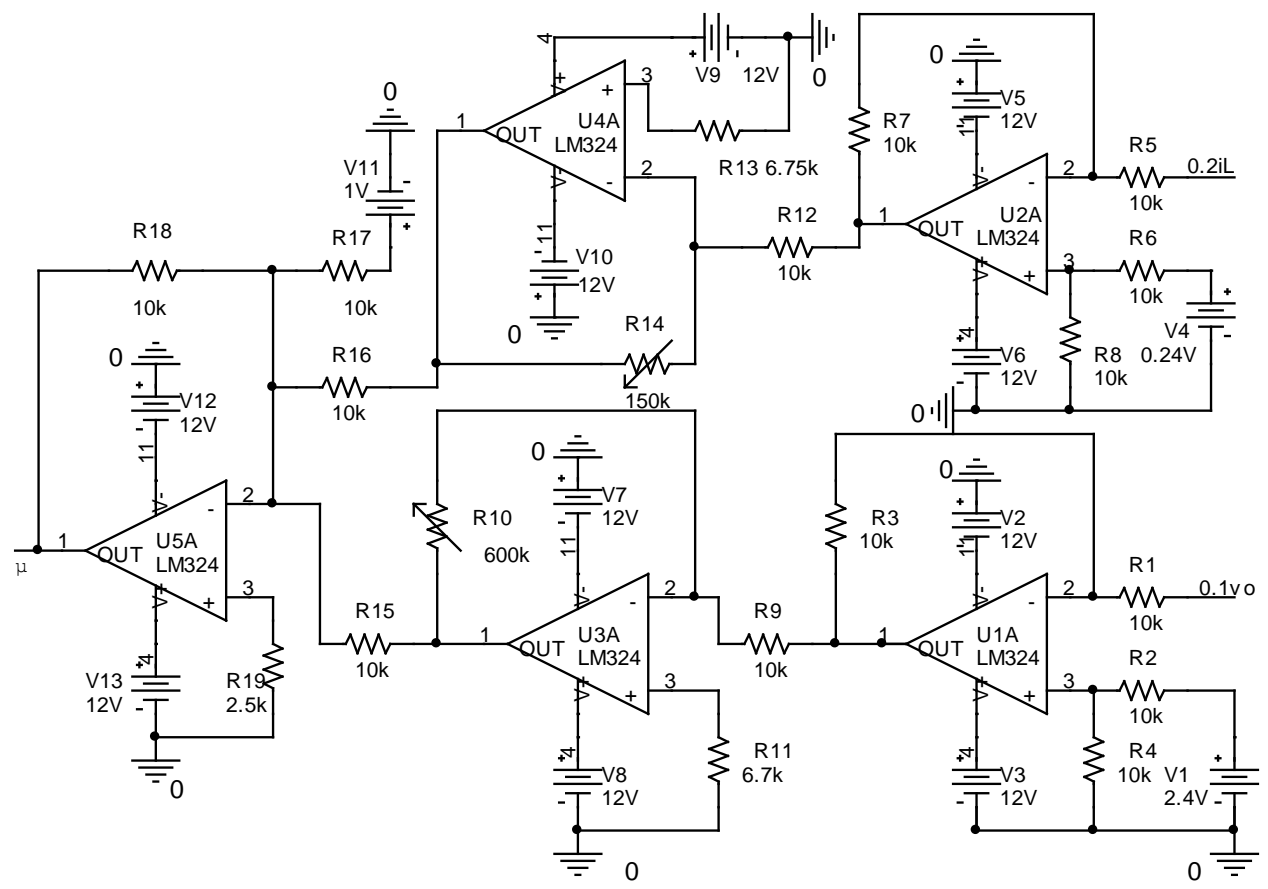

FIGURE III. CONTROL CIRCUIT

The dynamic performance of the buck converter controlled by the control circuit is simulated and analyzed. The parameters of the buck converter are chose as follows[13]: $L=1 \mathrm{mH}, \quad C=20 \mu \mathrm{F}, R=20 \Omega, E=48 \mathrm{~V}$. The PWM signal is modulated by triangle wave with frequency $100 \mathrm{kHz}$. Set the DC duty cycle 0.5 , then the operating point $\left(I_{\mathrm{d}}, V_{\mathrm{d}}\right)$ of buck converter is $(1.2 \mathrm{~A}, 24 \mathrm{~V})$. When the adjustable coefficient $k$ of proposed controller takes different values, the output voltage waveforms and the inductance current waveforms of the Buck converter are shown in Figure 4(a) (c) and Figure 5(a) (c), where the output voltages and the currents all can stabilize at the operating point and the transient responses are fast. With reducing $k$, the overshoot of the system will be reduced, but the steady state error will be lost in turn. Thus, the value of $k$ can be adjusted to get good dynamic quality in the range of tolerant steady state error.

Considering the load disturbance and input voltage disturbance, let $\mathrm{k}=5$ and the load resistance vary from $20 \Omega$ to $30 \Omega$, the input voltage vary from $48 \mathrm{~V}$ to $58 \mathrm{~V}$ at times of $0.8 \mathrm{~ms}$ and $1.4 \mathrm{~ms}$ respectively, the output voltage curve are obtained by PSpice simulation as seen in Figure 6 and Figure 7. It is seen that the proposed passive control strategy has good robustness to load disturbance and input voltage disturbance.

\section{CONCLUSIONS}

A passive control strategy for buck dc-dc converter is studied. The feedback controller is obtained by making the feedback equivalent system of buck converter passive. The proposed passive control can stability the output of buck converter with fast dynamic response speed and can effectively restrain disturbance of load and input voltage variation. By altering the controller parameter $k$, the dynamic performances of the buck converter can be adjusted.

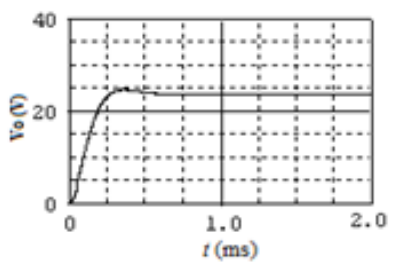

(a) $k=0.6$

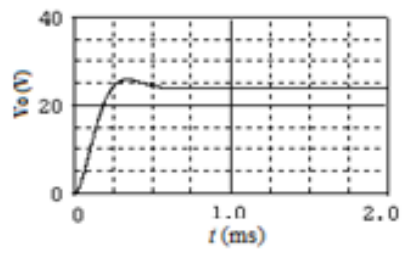

(b) $k=1$



(c) $k=5$ 


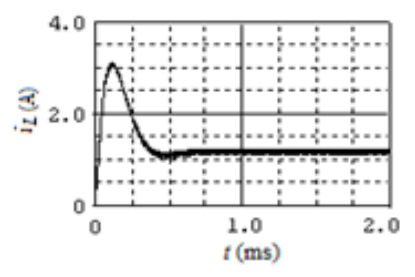

(a) $\mathrm{k}=0.6$

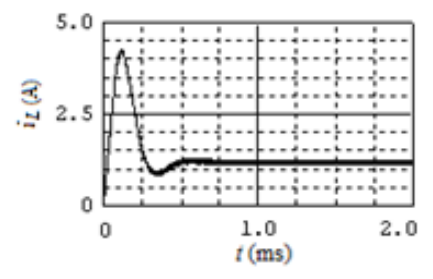

(b) $\mathrm{k}=1$



(c) $\mathrm{k}=5$

FIGURE V. INDUCTANCE CURRENT WITH DIFFERENT K

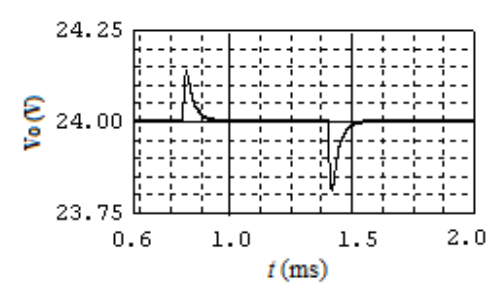

FIGURE VI. OUTPUT VOLTAGE WITH LOAD DISTURBANCE



FIGURE VII. OUTPUT VOLTAGE WITH INPUT VOLTAGE DISTURBANCE

\section{REFERENCES}

[1] G. Sandoval, H. Miranda, G. Espinosa-Pérez, V. Cárdenas, "Passivitybased control of an asymmetric nine-level inverter for harmonic current mitigation”, IET Power Electron., Vol. 5, no. 2, pp. 237-247, 2012.

[2] D. Noriega, G. Espinosa-Perez, V. Cardenas, J. Alvarez-Ramirez, "Passivity-based control of multilevel cascade inverters: high performance with reduced switching frequency", Int. J. Robust Nonlinear Control, Vol.20, no. 9, pp. 961-974, 2010.

[3] B. L. Zhang, J. H. Wang, F. J. Zhao, "Study on passivity-based control of TNPC PV grid-connected inverter”, Applied Mechanics and Materials, Vol.571 - 572, pp. 1000-1005, 2014.

[4] E. A. Oliveira, L. M. F. Morais, S. I. Seleme-júnior, "Power factor correction via passivity-based adaptive controller using buck converter operating in continuous mode", 11th Workshop on Control and Modeling for Power Electronics, Zurich, Switzerland, pp. 1-8, 2008.

[5] H. Z. Lu, J. H. Yang, J. M Yang., "Passivity analysis and adaptive control of brushless doubly-fed machines for adjustable speed drives", Control Theory \& Applications, vol. 26,no.12, pp: 1425 -1429, 2009. (in Chinese)
[6] C. Y. Chan, "Parallel damped passivity- based control of quasi-resonant converters”, IEE Proc. Electric Power Appl., vol.152, pp. 1093-1100, 2005.

[7] M. Masih, K. M. Mohammad, E. Mohammad, F. Mehrdad, M. Saeid, "Adaptive passivity-based control of a flexible-joint robot manipulator subject to collision”, Proceedings of the Institution of Mechanical Engineers, Part C: Journal of Mechanical Engineering Science, vol.229, no.5, pp. 840-849, 2015.

[8] M. Z. Andrea, L. Bakir, R. Paolo, "Passivity-based control of robotic manipulators for safe cooperation with humans”, International Journal of Control, vol.88, no.2, pp. 429-439, 2015.

[9] R. Leyva, A. Cid-pastor, C. Alonso, "Passivity-based integral control of a boost converter for large-signal stability”, IEE Proceedings. Control Theory and Applications, vol. 153, no.2, pp.139-146, 2006.

[10] A. Kwasinski, P. T. Krein, "Passivity- based control of buck converters with constant-power loads", IEEE 38th Annual Power Electronics Specialists Conference, Orlando, pp.259- 265, 2007.

[11] L. T. Wu, Z. H Yang., B. G. Xu, "Investigation of passivity-based control of DC/DC Converter", Transactions of China Electrotechnical Society, vol. 19, no. 4, pp. 66-69, 2004.(in Chinese)

[12] H. Sira-ramirez, R. A Perez-moreno, R. Ortega, "Passivity-based controllers for the stabilization of dc-to-dc power converters", Automatica, vol. 33, no. 4, pp. 499- 513, 1997.

[13] Z. Q. Wu, Z. J. Wang, "Kind of passivity control scheme for DC/DC converter”, Electric Machines and Control, vol.12, no.2, pp.147-150, 2008.(in Chinese)

[14] S. W. Mei, T. L. Shen, K. Z. Liu, Modern robust control theory and application, Beijing: Tsinghua University press, 2008, pp. 159-161. (in Chinese) 